EPJ Web of Conferences 47, 05007 (2013)

DOI: $10.1051 /$ epjconf/20134705007

(C) Owned by the authors, published by EDP Sciences, 2013

\title{
Benchmark low-mass objects in Moving Groups
}

\author{
M.C. Gálvez-Ortiz',2,a, M. Kuznetsov³, J.R.A. Clarke², Ya.V. Pavlenko ${ }^{3}$, \\ D.J. Pinfield ${ }^{2}$, H.R.A. Jones ${ }^{2}$, J.S. Jenkins ${ }^{4}$, J. Barnes ${ }^{2}$, B. Burningham ${ }^{2}$, \\ A.C. Day-Jones ${ }^{2,4}$, E.L. Martín ${ }^{1}$, A.E. García Pérez ${ }^{5}$ and R.S. Pokorny ${ }^{6}$
}

${ }^{1}$ Centro de Astrobiología (CSIC-INTA). Crta, Ajalvil km 4. 28850 Torrejón de Ardoz, Madrid, Spain

${ }^{2}$ Centre for Astrophysics Research, Science and Technology Research Institute, University of Hertfordshire, Hatfield AL10 9AB, UK

${ }^{3}$ Main Astronomical Observatory, Academy of Sciences of Ukraine, Golosiiv Woods, Kyiv-127, 03680, Ukraine

${ }^{4}$ Department of Astronomy, Universidad de Chile, Casilla Postal 36D, Santiago, Chile

${ }^{5}$ Department of Astronomy, University of Virginia, PO Box 400325, Charlottesville, VA 22904-4325, USA

${ }^{6}$ Yunnan Observatory, PO Box 110. CAS. 650011 Kunming, PR China

\begin{abstract}
In order to compile a sample of ultracool dwarfs that will serve as benchmarks for testing theoretical formation and evolutionary models, we selected low-mass cool (>M7) objects that are potentially members of five known young Moving Groups in the solar neighbourhood.

We have studied the kinematics of the sample, finding that 49 targets belong to the young disk area, from which 36 are kinematic member of one of the five moving groups under study. Some of the identified lowmass members have been spectroscopically characterised $\left(T_{e f f}, \log g\right)$ and confirmed as young members through a detailed study of age indicators.
\end{abstract}

\section{INTRODUCTION}

Identifying members of known moving groups (MG) or open clusters provides an important constraint on age and composition. While low-mass objects and brown dwarfs (BDs) evolutionary models are still pending of a good calibration at young age, compiling a sample of assessed members of any MG would allow the use of these objects as a test-bed for these models.

We assume the classical MG concept: a moving group is a young stellar population that shares a common space motion (e.g. [1]) whose members have a common origin - sharing age and composition. We focus in well documented groups: the Hyades supercluster (600 Myr), the Ursa Mayor group (Sirius supercluster) (300 Myr), IC 2391 supercluster (35-55 Myr), the Castor Moving Group (200 Myr), and the Local Association (20-150 Myr) or Pleiades moving group.

We present here a summary of the study and results of a major survey of ultra cool objects (UCDs) in young moving groups carried out in the last years. See [2], [3] and [4] for details.

\footnotetext{
${ }^{a}$ e-mail: mcz@cab.inta-csic.es
}

This is an Open Access article distributed under the terms of the Creative Commons Attribution License 2.0, which permits unrestricted use, distribution, and reproduction in any medium, provided the original work is properly cited. 
Table 1. Observing runs details.

\begin{tabular}{lllll}
\hline Date & Telescope & Instrument & Spect. range $(\AA)$ & Dispersion $(\AA)$ \\
\hline $28-30 / 12 / 06 \& 16-17 / 06 / 07$ & ESO-2.2m & FEROS $^{1}$ & $3500-9200$ & $0.033-0.081$ \\
$9 / 10 /-16 / 12 / 07$ & ESO-VLT-U2 & UVES $^{2}$ & $6650-10425$ & $0.027-0.041$ \\
$23 / 02-25 / 02 / 08$ & GEMINI & Phoenix $^{3}$ & $15532-15606$ & 0.074 \\
$28 / 03-21 / 06 / 08$ & ESO-VLT-U2 & UVES $^{2}$ & $6650-10425$ & $0.027-0.041$ \\
$01-09-2009-09 / 01 / 2010$ & ESO-VLT-U2 & UVES $^{2}$ & $5700-7530 \& 7650-9470$ & $0.027-0.041$ \\
$03-05 / 05 / 2010$ & 6.5 m Baade-Maguellan & IMACS Short-Camera $^{4}$ & $6550-10000$ & 1.98 \\
$15-16 / 02 / 2011$ & 6.5 m Baade-Maguellan & IMACS Short-Camera $^{4}$ & $4300-10800$ & 1.97 \\
\hline
\end{tabular}

${ }^{1}$ FEROS: The Fiber-fed Extended Range Optical Spectrograph; ${ }^{2}$ UVES: Ultraviolet and Visual Echelle Spectrograph.; ${ }^{3}$ Phoenix: PHOENIX Infrared high-resolution Spectrograph; ${ }^{4}$ IMACS: The Inamori Magellan Areal Camera and Spectrograph.

\section{SAMPLE SELECTION}

Sample A: Sample A was compiled by building a red catalogue of objects with proper motions in agreement with the MGs in study. We combined an extended version of the Liverpool-Edinburgh High Proper Motion survey (ELEHPM) and Southern Infrared Proper Motion Survey (SIPS) with the Two Micron All Sky Survey (2MASS), and applied colour cuts of $J-K_{s} \geq 1.0$ and $R-K_{s} \geq 5.0$ to select objects with spectral type predominantly later than M6. We also applied proper motion restriction, $\mu / \Delta \sigma_{1}>4$ ( $\mu$ is proper motion and $\sigma_{\mu}$ is the proper motion one sigma uncertainty) to select object with proper motion coherent with any of the MGs, ensuring proper motion accuracy. From the 817 red object catalogue built, by using these astrometric and photometric criteria, we obtained 132 objects possible members of one or more of the five MGs in study (see [2] for details).

Sample B: Sample B is formed by objects selected from [5] UCD catalogue containing 245 members of the southern Galactic plane, (covering 5042 sq.degs within $220^{\circ} \leq 1 \leq 260^{\circ}$ and $0^{\circ} \leq 1 \leq 60^{\circ}$ for $|\mathrm{b}| \leq 15^{\circ}$ ), identified as part of a deep search using 2MASS+SuperCOSMOS photometry and reduced proper motion constraints (see details in [6]). Applying the same selection process followed for sample A, [5] identified 23 objects as possible MG members, from which 13 form sample B.

High and low resolution spectra were obtained for sample A and sample B respectively. See Table 1 for details. Spectra were extracted using the standard reduction procedures in the IRAF ${ }^{1}$ twodspec/echelle package (bias subtraction, flat-field division, spectra extraction, telluric correction and wavelength and flux calibration). Wavelength calibration were obtained by a Th-Ar lamp spectra.

\section{KINEMATIC STUDY}

We obtained the objects' spectral types by using the spectral index PC3 as defined in [7] when possible, that provides an uncertainty of 0.5 subclass, or a combination of known spectral indexes if PC 3 was unusable, and $I-J$ colour index-spectral type relation from [8] for IR data (see [2]).

We then calculated a more accurate spectroscopic distance using a PC3-absolute J-magnitude relation from [9], that provides an error in distance of 12\%. For Phoenix data, distances were calculated from the absolute $\mathrm{J}$ magnitude-spectral type relation from [10].

Heliocentric radial velocities were also determined for sample A by applying the cross-correlation technique using the routine FXCOR in IRAF, (see e.g. [3]) or using an IDL routine (see e.g. [2]).

Using the obtained distances or any parallaxes from the literature, the proper motion data, and the calculated radial velocities, we computed the galactic space-velocity components $(U, V, W)$ of the sample by using the transformation matrices of [11]. In Fig. 1 left panel, we plot the UV plane for the

\footnotetext{
1 IRAF is distributed by the National Optical Observatory, which is operated by the Association of Universities for Research in Astronomy, Inc., under contract with the National Science Foundation.
} 

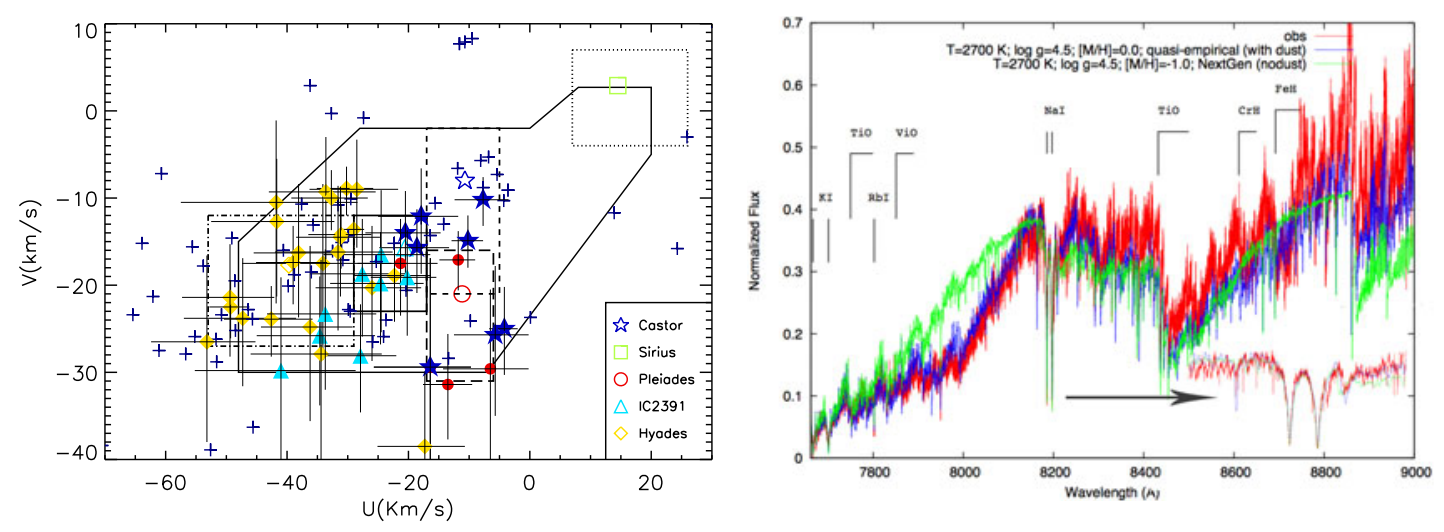

Figure 1. Left: UV space motion diagram. The YD boundaries are plot as a continuous line. The boxes indicate the expected kinematic range for the five MGs, whose centres are marked with large open symbols. Our kinematic members are shown as corresponding filled symbols. Right: example of model fitting and zoom on the Na I area.

whole sample including the boundaries (continuous line) that determine the young disk (YD) population as defined by [12]. We then classify objects as YD if they lie within the YD region in the UV plane (or if they could, given U and V $1 \sigma$ errors) and as kinematic MG member if they lie inside the relevant box (that marks expected kinematic range for each $\mathrm{MG}$ ) in each diagram, or having uncertainties that overlapped appropriately.

Applying this simple kinematic criterion to 68 targets of sample A, we found that 49 targets belong to the young disk area and that 36 of them possibly belong to one of the five MGs in the study.

\section{MODEL OF ATMOSPHERES AND SYNTHETIC SPECTRA}

In order to determine spectral characteristics of the targets $\left(T_{\text {eff }}, \log g\right)$ that help us in the age constraining, we compared a grid of generated synthetic spectra with the observations.

According to current concepts, dust plays an important role in the formation of late $\mathrm{M}$ dwarfs spectra (e.g. [13]). But the question about the effective temperature of stellar atmosphere above which dust effects can be neglected is still unclear. Literature studies suggest different temperature of transition between dust-free and dusty models, from $T_{e f f}=2500$ ([14]) to $T_{\text {eff }}=3000 \mathrm{~K}$ ([15]).

Therefore, synthetic spectra were computed for NextGen atmospheric model ([13]) without dust and semi-empirical models (from modified NextGen models) that include the effects of dust formation, by the WITA6 program ([16]). The grids were calculated for a $T_{\text {eff }}$ step of $100 \mathrm{~K}, \log g$ range of $4.0-5.5 \mathrm{~cm} \mathrm{~s}^{-2}$ with a step of $0.5 \mathrm{~cm} \mathrm{~s}^{-2}$ and metallicity of $[M / H]=0.0,-0.5$ and $-1.0 \mathrm{dex}$ for NextGen, and of $[M / H]=0.0$ for semi-empirical models. The best fit corresponds to the minimum value of the $S$-function described in [16], and is determined for each of the mentioned grid of models:

$$
S=\sum\left(f_{h} H_{\text {synt }}-H_{\text {obs }}\right)^{2}
$$

where $f_{h}$ is a normalisation parameter, $H_{s y n t}$ is the synthetic flux and $H_{o b s}$ is the observed flux.

The atmospheric structure of M8.5-M9.5 spectral type objects is very different from early M dwarfs. Since dust has a significant impact on the distribution of temperature vs. pressure in these atmospheres, more appropriate DUSTY and COND ([14]) models were used for the study of late M dwarfs. The DUSTY and COND models were also modified, in similar way to the model improvements for early $\mathrm{M}$ dwarfs. We introduced additional scattering of radiation by dust clouds and reduced the abundance of the molecules TiO and VO. The details of the method are described in [16] and [4]. 
Figure 1 right panel shows an example of the result in the fit of the red part of the spectra of a sample A object with a zoom-in in the $\mathrm{Na}$ I at $\sim 8190 \AA$ A region (bottom right).

\section{CHARACTERISATION AND AGE}

Although sample A is formed by objects that are already confirmed as kinematic members of MGs, determination of candidate's age can further constrain its group membership to a point of robustness. Sample B is formed by astrometric members and so further study should be apply to confirm any membership. This can be achieved in such ways as: using gravity sensitive spectroscopic features for objects that appear younger than $200 \mathrm{Myr}$; using the activity/age relation for late-type stars up to a spectral type of M7 (e.g. [17]); measuring the lithium 6708 A doublet (e.g. [19], [16]); and determining projected rotational velocities $(v \sin i)$ to differentiates between young and older M type objects (e.g. [18], [17] and based in Figures 9 and 10 of [17]).

We could measure $v \sin i$ for most of sample A. Surface gravity were obtained for sample A and B by the best model fitting. The presence of lithium and the equivalent widths of several gravity (Na I) and activity indicators $(\mathrm{H} \alpha)$ were measured also for both samples. Using the relation of these indicators with age, we estimated a range of ages that allowed us to confirm the targets of the two samples as young objects possible member of one of the MGs in study.

\section{RESULTS}

We confirmed up to now 16 objects as MG members. With known age and metallicity, the confirmed members will serve as testbeds for theoretical models. Also, determining the physical properties of brown dwarfs and very low-mass stars provides valuable information for our understanding of a broad range of topics including star and planet formation, circumstellar disks, dust formation in cool atmospheres, and the initial mass function.

\section{References}

[1] Pinfield D. J., Jones H. R. A., Lucas P. W., Kendall T. R., et al., MNRAS, 368, 1281 (2006)

[2] Clarke J. R. A., Pinfield D. J., Gálvez-Ortiz M. C., Jenkins J. S., et al., MNRAS, 402, 575 (2010)

[3] Gálvez-Ortiz M. C., Clarke J. R. A., Pinfield D. J., Jenkins J. S., et al., MNRAS, 409, 552 (2010)

[4] Gálvez-Ortiz M. C., Kuznetsov M. K., Clarke J. R. A., et al., MNRAS, in preparation, (2013)

[5] Folkes S. L., Phd thesis work, University of Hertfordshire (2009)

[6] Folkes S. L., Pinfield D. J., Jones H. R. A., Kurtev R., et al., MNRAS, 427, 3280F (20129

[7] Martín E. L., Delfosse X., Basri G., Goldman B., et al., AJ, 118, 2466 (1999)

[8] Leggett S. K., ApJS, 82, 351 (1992)

[9] Crifo F., Phan-bao N., Delfosse X., Forveille T., et al., A\&A, 441, 653 (2005)

[10] Dahn C. C., Harris H. C., Vrba F. J., Guetter H. H., et al., AJ, 124, 1170 (2002)

[11] Johnson D. R. H., Soderblom D. R., AJ, 93, 864 (1987)

[12] Eggen O. J., AJ, 89, 1358 (1984a)

[13] Hauschildt P., Allard F., ApJ, 512, 377 (1992)

[14] Allard F., Homeier D., Freytag B., ASPC, 448, 91A (2011)

[15] Jones H. R. A., Tsuji T., ApJ, 480, 1400 (1997)

[16] Pavlenko Ya. V., Jones H. R. A., Martín E. L., Guenther E., et al., MNRAS, 380, 3 (2007)

[17] Reiners A., Basri G., AJ, 684, 1390 (2008)

[18] Jenkins J. S., Ramsey L. W., Jones H. R. A., Pavlenko Y., et al., ApJ, 704, 975 (2009)

[19] Rebolo R., Martín E. L., Basri G., Marcy G. W., Zapatero-Osorio M. R., ApJ, 469L, 53R (1996) 